\title{
GW23-e1094 THE ASSOCIATION OF MCP-1 -2518 G/A POLYMORPHISM, ITS SERUM LEVELS WITH UNSTABLE ANGINA PECTORIS
}

doi:10.1136/heartjnl-2012-302920k.18

He Guo-ping, Jiang Jiu, He Guo-ping. Affiliated Wujin Hospital of Jiangsu University, Changzhou 213002, China

Objectives To investigate the effects of monocyte chemoattractant protein-1 (MCP-1) -2518 G/A genetic polymorphism on its serum levels and unstable angina pectoris (UAP), and the association of MCP-1 serum level with UAP in Chinese Han population of Sunan region.

Methods The -2518 G/A polymorphism of MCP-1 gene was genotyped in 203 patients with UAP and 192control subjects by PCR-RFLP and DNA sequencing; Serum concentration of MCP-1 was randomly measured in 72 patients with UAP and 73 control subjects by ELISA.

Results No significant difference was found in genotype distribution of the MCP-1-2518G/A between UAP and controls (all $p>0.05$ ), but $\mathrm{G}$ allele frequencies is significant lower in UAP group than that in controls $(p=0.044)$. Multivariate logistic regression analysis revealed that MCP-1-2518 G/A polymorphism was not associated with an increased risk of UAP ( $p>0.05)$. No significance was found in the serum level of MCP-1[(median/IOR) pg/ml] between genotypes of the MCP-12518G/A within UAP group and controls, respectively; The serum level of MCP-1 was significantly higher in UAP group (175.89/ $283.09 \mathrm{pg} / \mathrm{ml})$ than that in controls $(100.71 / 134.02 \mathrm{pg} / \mathrm{ml})(\mathrm{p}=0.007)$. Multiple linear regression analysis revealed that the serum levels of MCP-1 was associated with hypertension, diabetes mellitus, smoking and female in UAP group. Multivariate logistic regression analysis further revealed an elevated serum level of MCP-1 ( $>75$ th percentile) was associated with an increased risk of UAP [ $p=0.039$; OR 2.904 (1.058-7.970)].

Conclusions The serum level of MCP-1 was significantly higher in UAP group than that in controls, and an elevated serum level of MCP1 ( $>75$ th percentile) was associated with an increased risk of UAP in Chinese Han population of Sunan region; but the MCP-1-2518G/A polymorphism does not effect its serum levels nor contributes to an increased risk of UAP. 